\title{
WEAK COMPACTNESS OF MEASURES ${ }^{1}$
}

\author{
BENJAMIN B. WELLS, JR.
}

1. Introduction. This paper is concerned with a description of the weakly relatively compact subsets of the space of regular Borel measures on a compact Hausdorff space $X$. Several characterizations of such sets are known through the work of Pettis [6], Grothendieck [4], and Dieudonné [2]. We find a weak set of Boolean conditions on a family of open sets of $X$ to insure that convergence of a sequence of measures on each member of the family implies weak convergence of the sequence. This result is then applied to the Boolean algebra of regular open sets of $X$ to obtain a generalization to arbitrary compact Hausdorff spaces of a theorem of Grothendieck on Stonian spaces.

W. G. Bade has remarked that Grothendieck's theorem is equivalent to a well-known lemma of R. S. Phillips concerning the equivalence of weak convergence and weak-star convergence in $l_{\infty}^{*}$. Thus our generalization provides a new proof of Phillips' Lemma.

2. Preliminaries. Let $X$ be a compact Hausdorff space. Denote the Banach space of all real or complex-valued continuous functions on $X$ by $C(X)$, and denote the Banach space of all regular Borel measures on $X$ by $M(X)$. The dual space of $C(X)$ is $M(X)$, and if $\mu \in M(X)$ then $\|\mu\|=|\mu|(X)$, the total variation of $\mu$ on $X$. The topology for $M(X)$ of pointwise convergence on $C(X)$ is called the weak star topology and is denoted by $\sigma(M(X), C(X))$. The topology for $M(X)$ of pointwise convergence on $M(X)^{*}$, the dual of $M(X)$, is called the weak topology and is denoted by $\sigma\left(M(X), M(X)^{*}\right)$. From the Eberlein-Smulian Theorem we know that a subset $K$ of $M(X)$ is weakly relatively compact iff every sequence in $K$ has a weakly converging subsequence. Also useful is the fact that $M(X)$ is a weakly complete space. The classical necessary and sufficient condition for weak convergence of a bounded sequence $\left\{\mu_{n}\right\}$ in $M(X)$ is that $\lim _{n} \mu_{n}(E)$ exists for each Borel set $E \subseteq X$, [3, p. 308]. Two basic results in this connection are

THEOREM 1 (GRothendiecK [4, p. 147]). A sequence $\left\{\mu_{n}\right\}$ in $M(X)$ is $\sigma\left(M(X), M(X)^{*}\right)$-convergent iff for every sequence $\left\{E_{n}\right\}$ of pairwise disjoint open sets of $X \lim _{m} \mu_{n}\left(E_{m}\right)=0$ uniformly in $n$.

Received by the editors September 12, 1967.

1 This work is part of a Ph.D. thesis written under the direction of Professor W. G. Bade at Berkeley. It was supported by the National Science Foundation Grant GP. 5138. 
Theorem 2 (Dieudonné-Grothendieck). A sequence $\left\{\mu_{n}\right\}$ in $M(X)$ is convergent for the $\sigma\left(M(X), M(X)^{*}\right)$ topology iff for each open set $G \subseteq X, \lim _{n} \mu_{n}(G)$ exists.

Remark. Theorem 2 was first proved by Dieudonné [2] for $X$ metric and later by Grothendieck for $X$ an arbitrary compact Hausdorff space.

\section{Main results.}

Definition. Let $\beta$ be a family of Borel sets of $X$.

(a) We call $B$ a weak converging class for $M(X)$ provided every sequence $\left\{\mu_{n}\right\}$ in $M(X)$ which converges for each member $E$ of $B$ (i.e. $\left\{\mu_{n}(E)\right\}$ is a convergent sequence) converges for the weak topology.

(b) We call $B$ a bounding class for $M(X)$ provided every sequence $\left\{\mu_{n}\right\}$ in $M(X)$ which is bounded on each member $E$ of $B$ (i.e. $\left.\sup _{n}\left|\mu_{n}(E)\right|<\infty\right)$ is such that its sequence of norms $\left\{\left\|\mu_{n}\right\|\right\}$ is bounded.

Theorem 2 states that the family $B$ of open sets of $X$ is a weak converging class. Dieudonné also proved that it is a bounding class. Our first theorem gives a set of sufficient conditions on a family of open sets that it be both a weak converging class and a bounding class. We will apply our theorem to show that the regular open sets form a weak converging and a bounding class.

THEOREM 3. Let \& be a family of open sets of a compact Hausdorff space $X$, and let $\leftrightarrow$ satisfy

(1) $B$ is a basis for the topology of $X$;

(2) If $E_{1}$ and $E_{2}$ are in $B$, then $E_{1} \cap E_{2}$ is in $B$;

(3) If $E_{1}$ and $E_{2}$ are in $B$, and $\bar{E}_{1} \cap \bar{E}_{2}=\varnothing$, then $E_{1} \cup E_{2}$ is in $B$.

(4) If $K$ is compact, and $U$ is open, and $K \subseteq U$, then there exists an $E$ in $B$ such that $K \subseteq E \subseteq \bar{E} \subseteq U$;

(5) If $\left\{E_{n}\right\}$ and $\left\{G_{n}\right\}$ are sequences from B such that $E_{1} \subseteq E_{2} \subseteq \ldots$ $\subseteq E_{n} \cdots \subseteq G_{n} \subseteq \cdots G_{2} \subseteq G_{1}$, then there is some $E_{0}$ in $B$ such that $E_{n} \subseteq E_{0} \subseteq G_{n}$ for every $n$ ( $E_{0}$ is said to interpolate the sequences); then $B$ is a weak converging family for $M(X)$.

First we need the following

LEMma 1. Let $\left\{E_{n}\right\}$ be a sequence from a family of open sets $B$ satisfying conditions (1)-(5), and suppose that $\mathrm{Cl}\left(\cup_{i \neq n} E_{i}\right) \cap \bar{E}_{n}=\varnothing$ for each $n$. If $\nu$ is any nonnegative regular Borel measure, then for every $\delta>0$ there is an infinite set $A$ of positive integers and an $E_{A} \in B$ such that $\bigcup_{i \in A} E_{i} \subseteq E_{A}$ and $\nu\left(E_{A}\right)<\delta$. 
Proof. Let $\delta>0$ be given. We begin by choosing for each $n$ an open set $U_{2 n} \supseteq \bar{E}_{2 n}$ such that $U_{2 n} \cap \mathrm{Cl}\left(\bigcup_{i=1}^{\infty} E_{2 i+1}\right)=\varnothing$. Property (4) allows us to pick for each $n$ a set $F_{2 n} \in B$ such that $\left(\bar{E}_{2 n}\right)^{\prime} \supseteq F_{2 n} \supseteq U_{2 n}^{\prime}$. Note that

$$
\begin{aligned}
E_{1} \subseteq E_{1} \cup E_{3} \subseteq E_{1} \cup E_{3} \cup E_{5} \subseteq \cdots \subseteq \\
\cdots \subseteq F_{2} \cap F_{4} \cap F_{6} \subseteq F_{2} \cap F_{4} \subseteq F_{2} .
\end{aligned}
$$

By (5) there is some $G_{1} \in ß$ which interpolates the sequences; $G_{1}$ has the properties that $G_{1} \subseteq \bigcup_{i=1}^{\infty} E_{2 i+1}$ and $G_{1} \cap \bigcup_{i=1}^{\infty} E_{2 i}=\varnothing$.

If now $\nu\left(G_{1}\right)<\delta$ we are done. If $\nu\left(G_{1}^{\prime}\right)<\delta / 2$ then, since $G_{1}^{\prime}$ is compact and $\nu$ is a regular measure, it follows from (4) that there is some $F \in 囚$ such that $F \supseteq G_{1}^{\prime} \supseteq \bigcup_{i=1}^{\infty} E_{2 i}$, and is such that $\nu\left(F \cap G_{1}\right)<\delta / 2$. Hence

$$
\begin{aligned}
\nu(F) & =\nu\left(F \cap G_{1}^{\prime}\right)+\nu\left(F \cap G_{1}\right) \\
& =\nu\left(G_{1}^{\prime}\right)+\nu\left(F \cap G_{1}\right)<\delta / 2+\delta / 2=\delta
\end{aligned}
$$

and we would be done.

If neither $\nu\left(G_{1}\right)<\delta$ nor $\nu\left(G_{1}^{\prime}\right)<\delta / 2$, then we may repeat the above process to find disjoint subsequences $\left\{E_{n_{i}}\right\}$ and $\left\{E_{m_{i}}\right\}$ of the sequence $\left\{E_{2 n+1}\right\}$ and a $G_{2}$ in $B$ such that $G_{2} \supset \bigcup_{i=1}^{\infty} E_{n_{i}}, G_{2} \cap \bigcup_{i=1}^{\infty} E_{m_{i}}=\varnothing$, and $G_{2} \subseteq G_{1}$. If now $\nu\left(G_{2}\right)<\delta$ we would be done. If $\nu\left(G_{1} \cap G_{2}^{\prime}\right)<\delta / 2$, then we may pick an $H \in 囚$ such that $H \supseteq G_{2}^{\prime}$ and $\nu\left(H \cap G_{2}\right)<\delta / 2$. Note that

$$
G_{1} \cap H=\left(G_{1} \cap G_{2}^{\prime}\right) \cup\left(G_{2} \cap H\right) ;
$$

hence

$$
\nu\left(G_{1} \cap H\right)=\nu\left(G_{1} \cap G_{2}^{\prime}\right)+\nu\left(G_{2} \cap H\right)<\delta / 2+\delta / 2=\delta .
$$

We would be done since $G_{1} \cap H \in ß$ by (2), and $G_{1} \cap H \supseteq \bigcup_{i=1}^{\infty} E_{m_{i}}$.

If neither $\nu\left(G_{2}\right)<\delta$ nor $\nu\left(G_{1} \cap G_{2}^{\prime}\right)<\delta / 2$, then the above process may be repeated to get a $G_{3} \in ß, G_{3} \subseteq G_{2}$. If this process does not terminate we would be able to find a decreasing sequence $\left\{G_{n}\right\}$ in $B$ with the property that $\nu\left(G_{1}\right) \geqq \delta, \nu\left(G_{1}^{\prime}\right) \geqq \delta / 2 ; \nu\left(G_{2}\right) \geqq \delta, \nu\left(G_{1} \cap G_{2}^{\prime}\right) \geqq \delta / 2$; ... ; $\nu\left(G_{n}\right) \geqq \delta, \nu\left(G_{n-1} \cap G_{n}{ }^{\prime}\right) \geqq \delta / 2 ; \cdots$. However, the members of the sequence of sets $G_{1}^{\prime}, G_{1} \cap G_{2}^{\prime}, G_{2} \cap G_{3}^{\prime}, \cdots$ are pairwise disjoint. This would imply that the total variation of $\nu$ is infinite, which is a contradiction.

Proof of Theorem 3. Let $\left\{\mu_{n}\right\}$ be a sequence of regular Borel measures converging on each member of $B$. To show that $\left\{\mu_{n}\right\}$ is a Cauchy sequence for the weak topology, it would suffice to show that $\left\{\mu_{n}-\mu_{n+p_{n}}\right\}$ converges to 0 in the weak topology for each sequence 
$\left\{p_{n}\right\}$. Since $\left\{\mu_{n}(E)\right\}$ is convergent for each $E \in \mathbb{B}$, it follows that $\left\{\left(\mu_{n}-\mu_{n+p_{n}}\right)(E)\right\}$ converges to 0 for each $E \in B$. Hence, without loss of generality we may assume that $\left\{\mu_{n}\right\}$ is a sequence of regular Borel measures converging to 0 on each $E \in B$ and prove that $\left\{\mu_{n}\right\}$ is weakly convergent to zero.

Assume to the contrary that $\left\{\mu_{n}\right\}$ is not weakly convergent. Then by (4), regularity of the measures, and Theorem I.1 it follows that there is a sequence $\left\{E_{n}\right\}$ from $B$ and a positive $\epsilon$ such that $\mathrm{Cl}\left(\bigcup_{i \neq n} E_{i}\right)$ $\cap \bar{E}_{n}=\varnothing$ for each $n$, such that a subsequence of $\left\{\mu_{n}\right\}$, without loss of generality still called $\left\{\mu_{n}\right\}$, satisfies $\left|\mu_{n}\left(E_{n}\right)\right|>\epsilon>0$.

We now carry out an inductive process to obtain a subsequence $\left\{E_{n_{i}}\right\}$ of $\left\{E_{n}\right\}$ such that $\left\{\mu_{n}\right\}$ does not converge to zero on some

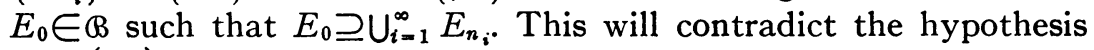
that $\left\{\mu_{n}\right\}$ converges to zero on every member of $B$.

We apply the lemma to the measure $\left|\mu_{1}\right|$ to get an infinite set of positive integers $A_{1}$ and an $E_{A_{1}} \in Q$ such that $\bar{E}_{A_{1}} \cap \bar{E}_{1}=\varnothing$ and $E_{A_{1}} \supseteq \bigcup_{n \in A_{1}} E_{n}$ and $\left|\mu_{1}\right|\left(E_{A_{1}}\right)<\epsilon / 3$.

First set $n_{0}=1$ and pick $n_{1} \in A_{1}$ so large that $\left|\mu_{n}\left(E_{1}\right)\right|<\epsilon / 3$ for all $n \geqq n_{1}$. Next apply the lemma again along with property (2) to extract an infinite set of positive integers $A_{2}$ from the set $\left\{A_{1} \cap\right.$ all integers

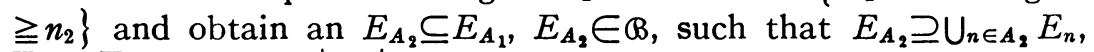
$\bar{E}_{A_{2}} \cap \bar{E}_{n_{1}}=\varnothing$, and $\left|\mu_{n_{1}}\right|\left(E_{A_{2}}\right)<\epsilon / 3$.

Now pick $n_{2}>n_{1}, n_{2} \in A_{2}$, so large that $\left|\mu_{n}\left(E_{1}\right)\right|+\left|\mu_{n}\left(E_{n_{1}}\right)\right|<\epsilon / 3$ for all $n \geqq n_{2}$. Continuing in this fashion we obtain a sequence of integers $\left\{n_{0}, n_{1}, n_{2}, \cdots\right\}$ and a decreasing sequence of sets in $\Theta, E_{A_{1}}$ $\supseteq E_{A_{2}} \supseteq E_{A_{3}} \supseteq \cdots$ such that the following hold:

$$
\begin{array}{ll}
\left|\mu_{n_{i}}\right|\left(E_{A_{i+1}}\right)<\epsilon / 3 & \text { for all } i, \\
\sum_{i=0}^{j-1}\left|\mu_{n}\left(E_{n_{i}}\right)\right|<\epsilon / 3 & \text { for all } n \geqq n_{j} .
\end{array}
$$

Consider the sequence of sets:

$$
\begin{aligned}
E_{1} \subseteq E_{1} \cup E_{n_{1}} \subseteq E_{1} \cup E_{n_{1}} \cup E_{n_{2}} \subseteq \cdots \\
\cdots \subseteq E_{A_{3}} \cup E_{n_{2}} \cup E_{n_{1}} \cup E_{1} \subseteq E_{A_{2}} \cup E_{n_{1}} \cup E_{1} \subseteq E_{A_{1}} \cup E_{1}
\end{aligned}
$$

Each member of the sequence is in $B$, and by property (5) we may choose an $E_{0}$ from $B$ which interpolates the sequence.

It simply remains to note that:

$$
\begin{aligned}
\left|\mu_{n_{j}}\left(E_{0}\right)\right| & \geqq\left|\mu_{n_{j}}\left(E_{n_{j}}\right)\right|-\sum_{i=1}^{j-1}\left|\mu_{n_{j}}\left(E_{n_{i}}\right)\right|-\left|\mu_{n_{j}}\right|\left(E_{A_{j+1}}\right) \\
& \geqq \epsilon-\epsilon / 3-\epsilon / 3=\epsilon / 3
\end{aligned}
$$


holds for every $j$. However, this contradicts our earlier assumption that the sequence $\left\{\mu_{n}(E)\right\}$ converges to 0 for each member $E$ of B. Q.E.D.

Corollary. If $B$ is a family of open sets of a compact Hausdorff space $X$ satisfying conditions (1)-(5) then $B$ is a bounding class for $M(X)$.

Proof. Suppose $\left\{\mu_{i}\right\}$ is a sequence in $M(X)$ satisfying $\sup _{i}\left|\mu_{i}(E)\right|$ $<\infty$ for every $E \in ß$. If $\left\{\left\|\mu_{i}\right\|\right\}$ is not bounded then without loss of generality we may drop to a subsequence and assume $\lim _{i}\left\|\mu_{i}\right\|$ $=\infty$. Now we may multiply each $\mu_{i}$ by an appropriate scalar (e.g. $\left.\left\|\mu_{i}\right\| 1 / 2 /\left\|\mu_{i}\right\|\right)$ to insure that $\lim _{i} \mu_{i}(E)=0$ for every $E \in ß$ while maintaining $\lim _{i}\left\|\mu_{i}\right\|=\infty$. The proof of the theorem shows that $\left\{\mu_{i}\right\}$ is $\sigma\left(M(X), M^{*}(X)\right)$-convergent to zero. However, this is impossible in view of $\lim _{i}\left\|\mu_{i}\right\|=\infty$. Q.E.D.

Definition. An open set $U$ is called regular if $U=\operatorname{int}(\bar{U})$.

The set of regular open sets of a topological space when ordered by set inclusion is a complete Boolean algebra. The supremum of a family $\left(U_{\alpha}\right)_{\alpha \in A}$ of regular open sets, denoted by $\mathrm{V}_{\alpha \in A} U_{\alpha}$, is defined to be int $\left(\mathrm{Cl}\left(\cup_{\alpha \in A} U\right)\right.$; the infimum, denoted by $\Lambda_{\alpha \in A} U_{\alpha}$, is defined to be $\operatorname{int}\left(\mathrm{Cl}\left(\bigcap_{\alpha \in A} U_{\alpha}\right)\right)$. The intersection of two regular open sets is regular. However, the union of two regular open sets need not be regular, and this fact presents the essential difficulty, since a Borel measure need not be even finitely additive with respect to the Boolean operations. However, if the closures of two regular open sets are disjoint then their union is regular. A complete discussion of regular open sets may be found in Halmos [5, p. 13].

THEOREM 4. If $X$ is a compact Hausdorff space and $B$ is the Boolean algebra of all the regular open sets of $X$, then $B$ is both a weak converging class and a bounding class.

Proof. \& obviously satisfies conditions (1)-(4) of Theorem 3. Also if $E_{1} \subseteq E_{2} \subseteq E_{3} \cdots \subseteq G_{3} \subseteq G_{2} \subseteq G_{1}$ is such that each member of the sequence is a regular open set, then both $\bigvee_{i=1}^{\infty} E_{i}$ and $\Lambda_{i=1}^{\infty} G_{i}$ interpolate the sequence. Thus $B$ satisfies the conditions of Theorem 3 , and we conclude that $B$ is both a weak converging class and a bounding class. Q.E.D.

Remark. W. G. Bade and P. C. Curtis had previously shown (unpublished) that the regular open sets are a bounding class.

Definition. A compact Hausdorff space $X$ is called Stonian if the closure of every open set is open. 
Lemma 2. $X$ is Stonian iff every regular open set is open and closed.

Proof. If $X$ is Stonian and $U$ is a regular open set, i.e., $U=\operatorname{int}(\bar{U})$, then $\operatorname{int}(\bar{U})=\bar{U}$ since $\bar{U}$ is an open set. Hence $U=\bar{U}$ and $U$ is open and closed. Conversely, if every regular open set is open and closed, then if $U$ is an open set, since $U \subseteq \operatorname{int}(\bar{U})$, it follows that $\bar{U} \subseteq \operatorname{int}(\bar{U})$ and hence that $\bar{U}=\operatorname{int}(\bar{U})$. Thus $\bar{U}$ is an open set. Q.E.D.

Our Theorem 4 is a generalization to arbitrary compact Hausdorff spaces of the following theorem of A. Grothendieck [4, p. 168].

Theorem 5. Let $X$ be Stonian and $\left\{\mu_{n}\right\}$ a sequence in $M(X)$. Then $\left\{\mu_{n}(E)\right\}$ is a convergent sequence for every open closed $E$ iff $\left\{\mu_{n}\right\}$ is convergent for the $\sigma\left(M(X), M(X)^{*}\right)$ topology.

Proof. By Lemma 2 the regular open sets of $X$ are precisely the open closed sets. Hence Theorem 4 gives us the result. Q.E.D.

Notation AND Definitions. Let $S$ be a discrete set. Then $\beta S$ denotes the Stone-Cech compactification of $S$. It is well known that $\beta S$ is a Stonian space. The space of all bounded real or complex-valued functions on $S$ with the supremum norm will be denoted by $B(S)$. The space of finitely additive measures on the field $\Sigma$ of all subsets of $S$ will be denoted by $b a(S, \Sigma)$. If $\mu \in b a(S, \Sigma)$ then $\|\mu\|=|\mu|(S)$, the total variation of $\mu$ on $S$. The atomic part of $\mu$ is defined by $\nu(E)$ $=\sum_{s \in E} \mu(s)$ where $E \in \Sigma$. We shall need the facts that $C(\beta S)$ is isometrically isomorphic to $B(S)$ and $M(\beta S)$ is isometrically isomorphic to $b a(S, \Sigma)$. For a complete discussion of these facts see Dunford and Schwartz [3, p. 311-313].

Grothendieck's proof of Theorem 5 was based on the following result due to Phillips [7].

THEOREM 6. Let $S$ be a discrete set and $\left\{\mu_{n}\right\}$ a sequence in ba $(S, \Sigma)$. If $\left\{\mu_{n}(E)\right\}$ converges to 0 for each $E \in \Sigma$, then $\left\{\left\|\nu_{n}\right\|\right\}$ converges to 0 , where $\nu_{n}$ is the atomic part of $\mu_{n}$.

REMARK (BADE). Theorem 5 is equivalent to Theorem 6.

Proof. Assume Theorem 5 and that $\left\{\mu_{n}\right\}$ is a sequence in $b a(S, \Sigma)$ such that $\lim _{n} \mu_{n}(E)=0$ for every $E \in \Sigma$. Let $\bar{\mu}_{n}$ be the correspondent of $\mu_{n}$ in $M(\beta S)$, and $k_{\bar{E}}$ be the correspondent of $k_{E}$ in $C(\beta S)\left(k_{E} \mathrm{de}-\right.$ notes the characteristic function of $E)$. Then $\bar{\mu}_{n}\left(k_{E}\right)=\mu_{n}\left(k_{E}\right)$, and it follows that $\bar{\mu}_{n}$ converges to 0 for each open closed set in $\beta S$. By Theorem $5\left\{\bar{\mu}_{n}\right\}$ converges to 0 in the $\sigma\left(M(\beta S), M(\beta S)^{*}\right)$ topology. Thus $\left\{\mu_{n}\right\}$ converges to 0 for the $\sigma\left(b a(S, \Sigma), b a(S, \Sigma)^{*}\right)$ topology.

Let $P$ denote the projection of norm 1 of $b a(S, \Sigma)$ onto $l_{1}(S)$ defined 
by $P: \mu \rightarrow \nu$ where $\nu$ is the atomic part of $\mu . P$ is norm continuous and hence is continuous for the weak topologies. Thus $\left\{P \mu_{n}\right\}=\left\{\nu_{n}\right\}$ converges to 0 for the $\sigma\left(l_{1}(S), l_{\infty}(S)\right)$ topology. By a theorem of Banach $\left[1\right.$, p. 137] $\left\{\left\|\nu_{n}\right\|\right\}$ converges to zero.

Assume Theorem 6 and that $\left\{\mu_{n}\right\}$ is a sequence of regular Borel measures on a Stonian space $S$ which converges to 0 on each open closed subset of $S$. To show that $\left\{\mu_{n}\right\}$ is weakly convergent to 0 , it suffices to show (by Theorem 1 ) that $\left\{\mu_{n}\left(E_{n}\right)\right\}$ converges to 0 , where $\left\{E_{n}\right\}$ is an arbitrary sequence of pairwise disjoint open closed subsets of $S$.

Define for each $n$ a set function $\nu_{n}$ on $N$, the set of positive integers:

$$
\nu_{n}(A)=\mu_{n}\left(\bigvee_{i \in A} E_{i}\right) \text { where } A \subseteq N .
$$

Note that $\nu_{n}$ is bounded and finitely additive, and hence an element of $b a(N, \Sigma)$. Since $\left\{\mu_{n}\right\}$ converges to 0 on each open closed subset of $S,\left\{\nu_{n}(A)\right\}$ converges to 0 for each $A \in \Sigma$. Theorem 6 allows the conclusion that $\lim _{n} \sum_{i=1}^{\infty}\left|\nu_{n}(i)\right|=0$. In particular

$$
\lim _{n}\left|\nu_{n}(n)\right|=\lim _{n}\left|\mu_{n}\left(E_{n}\right)\right|=0 \text {. Q.E.D. }
$$

The proof of Theorem 4 thus provides a new proof of Theorem 6 .

\section{REFERENCES}

1. S. Banach, Theorie des opérations linéaires, Monogr. Mat., Warsaw, 1932.

2. J. Dieudonné, Sur la convergence des suites de measures de radon, An. Acad. Brasil. Ci. 23 (1951), 21-38, 277-282.

3. N. Dunford and J. Schwartz, Linear operators. Part I: General theory, Interscience, New York, 1964.

4. A. Grothendieck, Sur les applications linéaires faiblement compact d'espaces $d u$ type $C(K)$, Canad. J. Math. (1953), 129-173.

5. P. Halmos, Lectures on Boolean algebras, Van Nostrand Math. Studies, Princeton, N. J., 1963.

6. B. J. Pettis, On integration in vector spaces, Trans. Amer. Math. Soc. 44 (1938), 277-304.

7. R. S. Phillips, On linear transformations, Trans. Amer. Math. Soc. 48 (1940), 516-541.

University of California, Berkeley 